\title{
Innovaciones docentes mediante aprendizaje basado en problemas en Fisioterapia General
}

\section{Teaching innovations through problem-based learning in General Physiotherapy}

ANTONIO SÁNCHEZ GONZÁLEZ

ORCID: https://orcid.org/0000-0002-1206-9334

Universidad de Sevilla

Facultad de Enfermería, Fisioterapia y Podología

Departamento de Fisioterapia

Correo electrónico: asanchez35@us.es

Fecha de recepción: 17-06-2019

Fecha de aceptación: 01-07-2019

DOI: http://dx.doi.org/10.12795/9788447221912.043

Pp.: $978-1000$ 


\section{Resumen:}

El Ciclo de Mejora en el Aula (CIMA), ha sido aplicado en la asignatura de Fisioterapia General del Grado en Fisioterapia. Después de analizar la estructura de los contenidos de esta asignatura, hemos integrado los temas teórico-prácticos, incluyendo algunas mejoras de innovación docente, para conseguir la construcción del conocimiento conforme a las diferentes formas de aprendizaje del alumnado. Hemos intentado conseguir que los estudiantes adquieran las competencias definidas en el programa de la asignatura, empleando un modelo didáctico de aprendizaje basado en problemas, introduciendo distintos sistemas de gamificación que nos permitiesen implementar el CIMA, para alcanzar las habilidades y competencias, así como la calidad docente. Para evaluar todo el proceso hemos utilizado un cuestionario previo de conocimientos, desarrollando el material teórico-práctico y planteando un problema o reto por parejas que sería evaluado mediante una rúbrica. A continuación, reflexionamos sobre el modelo de aprendizaje de forma grupal y finalizamos con una evaluación global del proceso. Dentro de estas evaluaciones también se valoró el grado de satisfacción docente por parte del alumnado. Analizados los resultados hay que destacar el ascenso en el grado de adquisición de conocimientos de los estudiantes. Las valoraciones del ambiente en clase, de sus compañeros y de las instalaciones, asi como de los materiales empleados que fueron entre el $75 \%$ y el $100 \%$, y la valoración del docente y del resto del personal por encima de $75 \%$, lo que demuestra unos valores muy significativos.

Palabras Claves: Fisioterapia general, Grado en Fisioterapia, Docencia Universitaria, Experimentación Docente Universitaria, Aprendizaje Basado en Problemas.

Jornadas de Formación e Innovación Docente del Profesorado | № 2 (2019) Esta obra se distribuye con la licencia Creative Commons Reconocimiento-NoComercial-SinObraDerivada Internacional (CC BY-NC-ND 4.0.) 


\section{Abstract:}

The current cycle of improvement in the classroom (CIMA) has been applied in the subject of General Physiotherapy, of the Degree in Physiotherapy. After analyzing the structure of the contents of this subject, we have integrated the theoretical-practical topics, including some improvements of teaching innovation to achieve the construction of knowledge and according to the different ways of learning of the students. We have tried that students acquire the competences defined in the program of the subject, using a didactic model of problem-based learning and introducing different gamification systems that allow us to implement the CIMA, to achieve the skills and competences, as well as the teacher quality. To evaluate the process we have used a previous questionnaire of knowledge, developing theoretical-practical material and posing a problem or challenge for couples that would be evaluated by a rubric. Next, we reflect on the learning model in group and finish with a global evaluation of the process. Within these evaluation, the degree of teacher satisfaction on the part of the students was also assessed Analyzed the results, we must highlight the rise in the degree of knowledge acquisition of students. Valuations of the classroom environment, of their classmates and of the facilities, as well as of the materials used, were between $75 \%$ and $100 \%$, and the evaluation of the teacher and the rest of the staff were above $75 \%$, which shows very significant values.

Keywords: General Physiotherapy, Physiotherapy Degree, University Teaching, University Teaching Experience and Problem-based learning.

Jornadas de Formación e Innovación Docente del Profesorado | № 2 (2019) Esta obra se distribuye con la licencia Creative Commons 


\section{Breve descripción del contexto:}

La experiencia innovadora que se presenta se ha realizado en el primer curso del Grado de Fisioterapia, en la asignatura obligatoria de Fisioterapia General (FG), algo radicalmente diferente del ciclo anterior que se implementó con alumnos/as de cuarto curso. Esta materia cuenta con 6 créditos ECTS y se distribuye en 30 horas teóricas y 30 horas de prácticas de laboratorio. El Ciclo de Mejora en el Aula (en adelante CIMA), se realizó en las prácticas de laboratorio, en un grupo de 10 alumnos/as, realizando una sesión de 2 horas de forma semanal y en distintas partes del temario (concretamente en las prácticas ubicadas desde la semana 4 a la semana 10). El CIMA se ha llevado a cabo a lo largo de 14 horas teórico-prácticas, lo que ha supuesto el desarrollo de la red de contenidos programados para este periodo. Previo a la implementación de este plan de innovación, hemos tenido en cuenta las experiencias realizadas en anteriores cursos académicos, aunque se dieron en distintas asignaturas y cursos del mismo grado, siempre en materias obligatorias e incluso en otro centro universitario. Como aspecto relevante, hay que destacar que el ciclo actual se ha aplicado por primera vez en esta asignatura y se ha desarrollado con alumnos/as de primero del Grado en Fisioterapia. Se ha diseñado una proporcionada planificación de las actividades a realizar con el propósito de encontrar el menor número de obstáculos posibles durante todo el proceso.

Jornadas de Formación e Innovación Docente del Profesorado | № 2 (2019) Esta obra se distribuye con la licencia Creative Commons 


\section{Diseño previo del CIMA.}

\section{Mapa de contenidos, problemas y núcleos de trabajo en el aula}

Con respecto al mapa de contenidos hemos incluido preguntas estructurantes o preguntas clave que proponían un reto intelectual o problema a resolver por los alumnos/ as. Por otro lado, se han propuesto subpreguntas, con el objetivo de abarcar los contenidos conceptuales, los procedimentales y actitudinales, enlazando unos con otros y generando un entramado que incitase al alumnado a buscar las respuestas apropiadas a dichas preguntas.

Organizamos los contenidos de acuerdo con la lógica de la disciplina: relación, jerarquía, y respuesta de problemas, entre otros aspectos. Consideramos que dar con una buena pregunta clave es fundamental, ésta debe ser motivadora para el estudiante y el problema a resolver debe suponer un reto. Por otra parte, también tenemos que descomponer el problema principal en subproblemas con sus propios contenidos conceptuales y jerarquizados. No debemos olvidar que nos encontramos con alumnos/as de primer curso sin una base sólida de conocimientos en la disciplina. Con las respuestas a estas preguntas ofrecemos un hilo conductor al aprendizaje integral de toda la materia. Lo que se pretende, en definitiva, es que contesten a estas cuestiones reflejadas en el mapa conceptual del tema, que en esta ocasión ha sido Introducción a la Masoterapia, dentro de la asignatura de FG, tal como se puede observar en la figura 1. 


\section{ANTONIO SÁNCHEZ GONZÁLEZ}

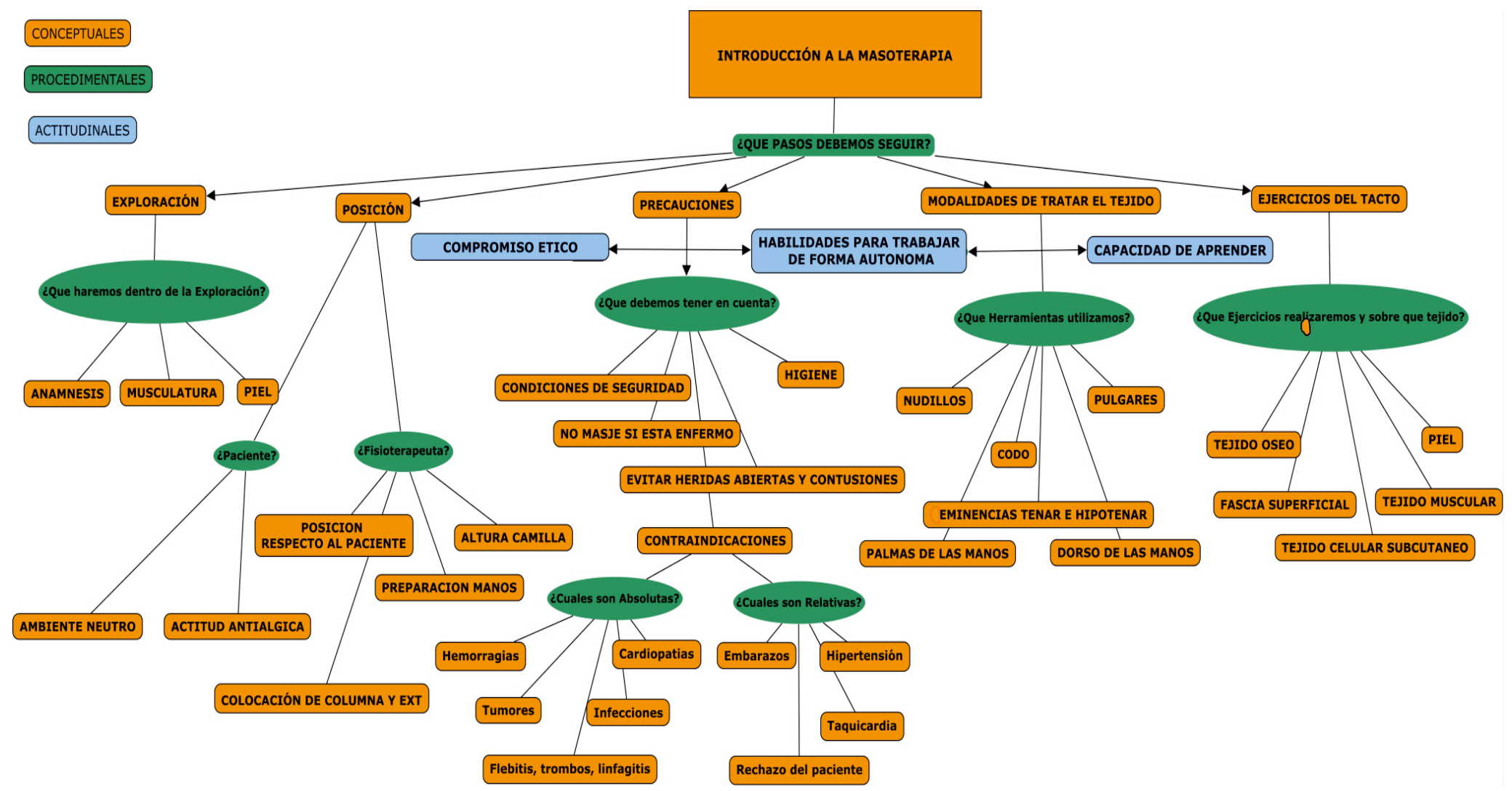

Figura 1. Mapa de contenidos Introducción a la Masoterapia. Fuente: Elaboración propia.

Jornadas de Formación e Innovación Docente del Profesorado I № 2 (2019)

cC (i) $\&$ Esta obra se distribuye con la licencia Creative Commons

cc)

Internacional (CC BY-NC-ND 4.0.) 
Para la elaboración de los mapas de contenidos hemos aplicado conocimientos aprendidos del Curso General de Docencia Universitaria (2015-2016) así como de las conclusiones derivadas de las Jornadas FIDOP 2018 y del Manual de Enseñanza Universitaria (Porlán, 2017).

En todo este proceso no podemos obviar la necesaria adaptación de la materia a los niveles de conocimiento de los estudiantes, como avanzábamos anteriormente, adecuando nuestra propuesta de contenidos al nivel previo obtenido mediante los datos recogidos en el pretest del cuestionario de ideas iniciales. Como sabemos, se trata de estudiantes de primer curso, para quienes todo es nuevo $y$, generalmente, nos encontramos con que tienen un conocimiento muy limitado o escaso de la disciplina. También hay que destacar las altas expectativas y entusiasmo por aprender con la que asisten a las clases, pudiéndose ser este dato un factor positivo en el proceso de enseñanza-aprendizaje.

\section{Modelo metodológico posible y secuencia de actividades:}

Se propone una experiencia metodológica inspirada en el aprendizaje basado en problemas (ABP). Como se sabe, esta metodología está centrada en el aprendizaje, la investigación y la reflexión que debe aplicar el alumnado para resolver un problema planteado por el docente. Por lo tanto, aplicaremos un modelo de aprendizaje a través del trabajo autónomo y en equipo, con un compromiso fuerte y real con el aprendizaje colaborativo. Además, se pretende que sean capaces de interrelacionar distintas materias o disciplinas académicas.

Para diseñar esta metodología hemos aprovechado todo lo aprendido en el Curso de Nuevas Metodologías para el Aprendizaje Activo de los Estudiantes (2018). Hemos 
pretendido, en todo momento, integrar las clases teórico-prácticas partiendo del trabajo en equipo sin olvidar el propio trabajo individual, con el objetivo de mejorar la calidad de la docencia y el proceso enseñanza-aprendizaje. De este modo, "los estudiantes pasan a ser sujetos activos de su aprendizaje", construyendo el conocimiento sobre la base del problema planteado y resuelto por ellos mismos (Guerra, 2009).

El método de ABP representa un flujo de desarrollo en la experiencia de aprendizaje (figura 2), siendo la motivación y la implicación del estudiante, como hemos dicho anteriormente, un elemento clave para fomentar el aprendizaje activo de este.

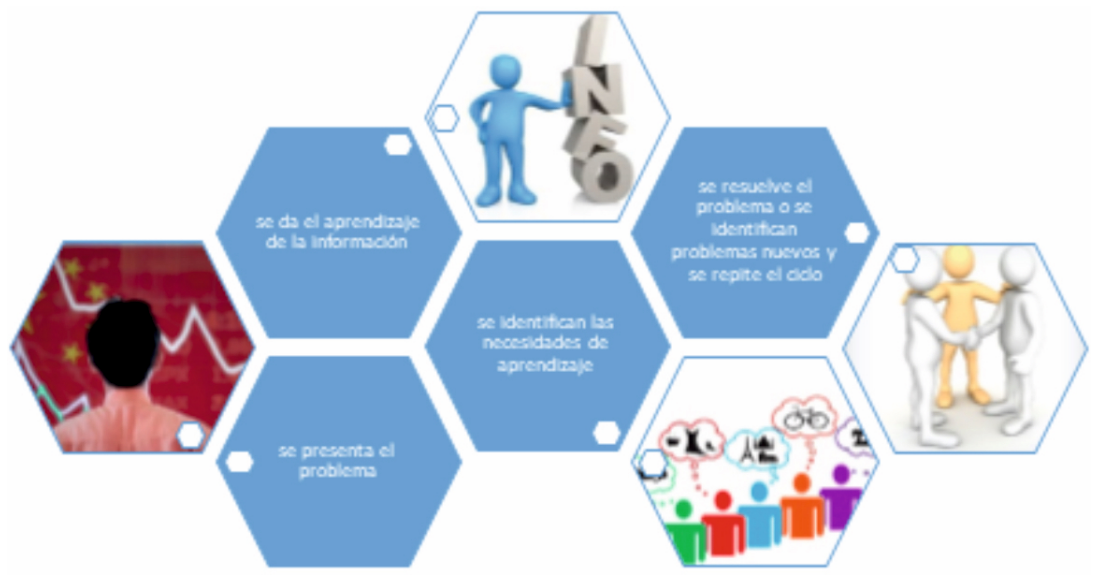

Figura 2. Flujo de desarrollo en la experiencia de Aprendizaje (Fuente: elaboración propia)

Iniciamos las clases en esta materia siguiendo un modelo tradicional de clase magistral, similar a la figura 3, y más adelante, a partir de la práctica número cuatro, comenzamos a profundizar en el contexto de aprendizaje: tipo de alumnos, relación existente entre ellos, afinidades y conocimientos sobre la materia, entre otros, para más adelante poder aplicar el modelo metodológico propuesto. 


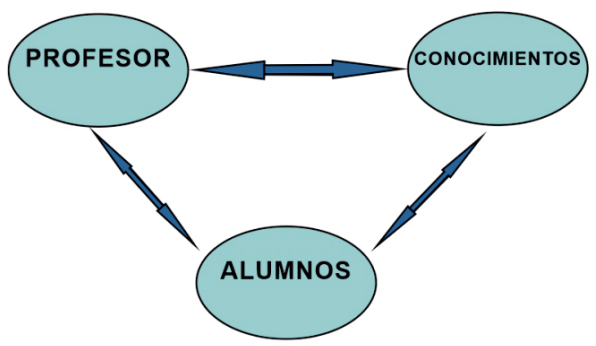

ACTUAL

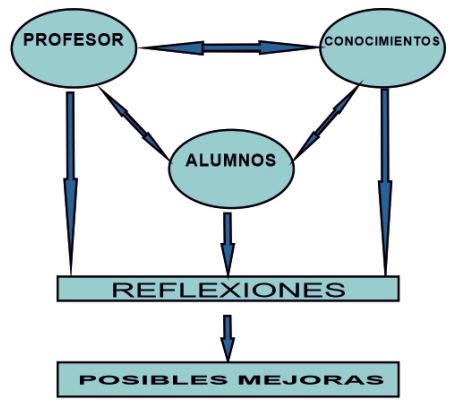

PROPUESTO

Figura 3. Modelo metodológico actual y modelo propuesto. Fuente: Elaboración propia.

Con relación a las limitaciones del modelo metodológico actual, consideramos que éste no se encuentra en consonancia a estos tiempos y, sobre todo, con el sistema de Bolonia. Por ello, creemos necesario y oportuno modificar el modelo tradicional con el modelo propuesto, donde adquiere protagonismo una metodología activa y participativa, incluyendo actividades didácticas innovadoras que favorecen el proceso de enseñanza-aprendizaje del alumnado. Nos centraremos en la aplicación de la teoría, la práctica y la discusión con los estudiantes (reflexiones), y tendremos en cuenta todas sus sugerencias y propuestas, que sin duda aportan frescura e implicación en todo el desarrollo del aprendizaje.

Por otro lado, entendemos que este planteamiento es mucho más flexible, permitiéndonos incorporar las modificaciones que creamos oportunas, según el avance de las clases.

A continuación, presentamos la secuencia de actividades en un tema específico, siendo esta dinámica la misma que se sigue en las respectivas clases que han sido sometidas a Ciclo de Mejora en el Aula: 
Tabla 1. Secuencia de actividades del CIMA de la asignatura FG

\begin{tabular}{|c|c|c|c|c|}
\hline $\begin{array}{c}\text { Teoria y } \\
\text { grupo }\end{array}$ & Horario & Actividades a desarrollar & $\begin{array}{l}\text { Tiempo } \\
\text { presencial }\end{array}$ & MM \\
\hline \multirow{6}{*}{$\begin{array}{l}\text { Tema } 4 . \\
\text { Grupo } 7\end{array}$} & \multirow{3}{*}{$\begin{array}{l}12: 30 \\
-13: 30\end{array}$} & $\begin{array}{l}\text { 1. Presentación y explicación del } \\
\text { procedimiento }\end{array}$ & $10 \mathrm{~min}$ & $\mathrm{~T}$ \\
\hline & & $\begin{array}{l}\text { 2. Cuestionario Previo individual } \\
\text { (Kahoot, Socrative, etc.,) }\end{array}$ & $10 \mathrm{~min}$ & $\mathrm{~T}-\mathrm{P}$ \\
\hline & & $\begin{array}{l}\text { 3. Desarrollo y exposición del } \\
\text { material teórico }\end{array}$ & $30 \mathrm{~min}$ & $P$ \\
\hline & \multirow{3}{*}{$\begin{array}{l}13: 30 \\
-14: 30\end{array}$} & $\begin{array}{l}\text { 1. Realizar todas las técnicas, } \\
\text { introducir reto por parejas }\end{array}$ & $40 \mathrm{~min}$ & $P$ \\
\hline & & $\begin{array}{l}\text { 2. Realizar las Reflexiones en } \\
\text { grupo }\end{array}$ & $10 \mathrm{~min}$ & $\mathrm{R}$ \\
\hline & & $\begin{array}{l}\text { 3. Evaluaciones: Individuales, } \\
\text { grupo, actividad y Profesor }\end{array}$ & $20 \mathrm{~min}$ & $\mathrm{R}$ \\
\hline
\end{tabular}

En primer lugar, antes de iniciar la impartición de los contenidos, hemos pasado en todas las clases un cuestionario inicial que nos permitiese conocer el nivel de conocimiento que tenía el alumnado sobre la materia.

Seguidamente, comenzamos el desarrollo del programa teórico-práctico partiendo de la pregunta principal o pregunta inicial, por ejemplo, en el caso de la introducción a la masoterapia, sería ¿qué pasos debemos seguir?. Una vez impartida toda la materia teórica junto con la práctica y, habiendo promovido la participación activa de los estudiantes en la elaboración de los conceptos, introdujimos experiencias profesionales. Aquí fue donde utilizamos otros conocimientos adquiridos con anterioridad del curso Como Implicar a los Estudiantes a Través de Nuevas Herramientas Tecnológicas (2018).

Jornadas de Formación e Innovación Docente del Profesorado | № 2 (2019) Esta obra se distribuye con la licencia Creative Commons Reconocimiento-NoComercial-SinObraDerivada Internacional (CC BY-NC-ND 4.0.)

Internacional (CC BY-NC-ND 4.0.) 
Durante todo el desarrollo docente hemos ido introduciendo una serie de subpreguntas como puede ser el siguiente ejemplo: ¿qué haremos dentro de la exploración?, para que vayan dando forma a toda la estructura de aprendizaje. Una vez terminada esta etapa, pasamos a implementar todo lo aprendido en la práctica por parejas. En este momento formulamos pequeños problemas o retos, para que ellos intentaran resolverlos en la propia práctica, siempre con el apoyo del docente y con las aportaciones de los compañeros. Durante esta fase, surgieron nuevas preguntas y dudas que se profundizaron en la etapa de reflexiones grupales.

En la tabla 2, recogemos reflexiones textuales derivadas de la fase metodológica de nuestra propuesta de modelo metodológico.

Tabla 2. Reflexiones del alumnado sobre los contenidos de la asignatura FG

Reflexión A
“Las prácticas de fisioterapia general nos parecen muy interesantes y
útiles ya que en cada clase aprendemos muchas cosas nuevas, pero
en la parte teórica explicada en la pizarra nos parece muy extensa y
se hace demasiado rápida para poder tomar apuntes y poder realizan
después un buen cuaderno de la asignatura”.
Reflexión B
"En general, el grupo se encuentra muy contento por la decisión que
se tomó la última clase de ampliar el tiempo de prácticas y todos es-
tamos de acuerdo en que la clase se ha ido desarrollando de forma
positiva. Sin embargo, seguimos teniendo el inconveniente de que el
número de alumnos es impary, por tanto, siempre habrá un grupo de
tres a la hora de llevar a cabo la parte práctica, lo que puede perju-
dicar el aprendizaje. Ante este problema, el profesor nos propone in
rotando para que así no sean siempre las mismas personas las que
compongan el grupo de tres. Por último, se propone al profesor un

Jornadas de Formación e Innovación Docente del Profesorado | № 2 (2019) Esta obra se distribuye con la licencia Creative Commons Reconocimiento-NoComercial-SinObraDerivada 4.0 Internacional (CC BY-NC-ND 4.0.) 
mayor tiempo para poder anotar la parte teórica, el cual nos explica cómo debemos tomar apuntes y nos recuerda del poco tiempo que disponemos para realizar cada parte que compone la clase de cada semana".

Reflexión C

"De forma generalizada, la clase se encuentra satisfecha con el desa rrollo de la práctica. Algunos alumnos destacamos nuestra aprobación con respecto a la duración de la parte práctica, mayor que en la clase anterior".

Reflexión D

"La práctica ha ido bastante bien ya que hemos estado la mayor parte del tiempo realizando la parte práctica. El único inconveniente es que la parte teórica va demasiado rápido porque se supone que lo sabemos de las clases teóricas, pero vamos muy atrasados y no lo entendemos".

Reflexión E

"La práctica a todos nos ha parecido muy bien, además de notar una mejoría en cuanto al tiempo práctico para el masaje".

Esta metodología ha aportado una mejora sustancial a la hora de encontrar soluciones a los problemas planteados, ya fuese por las aportaciones de los estudiantes o los ejemplos de la propia experiencia profesional. Hemos de subrayar que esta faceta del aprendizaje ha sido muy satisfactoria para el alumnado a la luz de los comentarios vertidos por ellos.

Durante el trascurso de la implementación hemos ido introduciendo metodologías innovadoras propias de la era digital, sin olvidar la utilización de diversas herramientas de gamificación, con aplicaciones como Socrative (para realizar los pretest y los postest).

Jornadas de Formación e Innovación Docente del Profesorado | № 2 (2019) Esta obra se distribuye con la licencia Creative Commons 
Sobre la utilización de estas aplicaciones Sánchez y Quintero subrayan que "el uso de Socrative representa una metodología innovadora que rompe con el método de clase tradicional, creando un espacio más dinámico y participativo, favoreciendo una gran implicación y atención en el proceso de enseñanza-aprendizaje" (Sánchez y Quintero, 2018)

Un elemento esencial que no podemos olvidar es la evaluación del proceso de enseñanza-aprendizaje. Para ello hemos utilizado distintas evaluaciones continuas, feedforward, rúbricas de evaluación, diarios reflexivos y también hemos recogido las opiniones de los estudiantes.

\section{Cuestionario inicial y final para la evaluación del proceso de enseñanza-aprendizaje:}

Hemos diseñado cuestionarios iniciales pretest, mediante la aplicación Socrative (tabla 3), con la finalidad de analizar el punto de partida de cada estudiante, así como el grado de conocimiento sobre los contenidos a impartir. Con la misma aplicación y una vez finalizada la práctica volvemos a realizar un cuestionario final (postest) para determinar el grado de aprendizaje adquirido por cada estudiante, con objeto de poder analizar el escalón en el que cada uno se encontraba. Con ello, se ha pretendido analizar las necesidades de cada discente y reflexionar sobre qué aspectos de nuestra docencia debe ser modificado para conseguir vencer el bloqueo y permitir la adquisición del nivel más elevado de la escalera, por parte del alumnado. 
Tabla 3. Cuestionario diseñado para la asignatura FG. Ejemplo del Tema 4

\begin{tabular}{|c|l|}
\hline Pregunta & \\
\hline 1 & ¿De cuantas fases se compone el masaje clásico? \\
\hline 2 & Describe los pasos previos al masaje \\
\hline 3 & $\begin{array}{l}\text { El masaje de roce superficial ¿dentro de que bloque de los } \\
\text { siguientes se encuentra? }\end{array}$ \\
\hline 4 & $\begin{array}{l}\text { Dentro de los bloques en los que se divide el masaje clásico } \\
\text { ¿Cuál tiene más maniobras? }\end{array}$ \\
\hline 5 & ¿Cómo se denomina también el masaje superficial? \\
\hline 6 & $\begin{array}{l}\text { Al masaje de amasamiento ¿también se le denomina } \\
\text { petrissación? }\end{array}$ \\
\hline 7 & $\begin{array}{l}\text { El procedimiento de percusión tiene un efecto profundo y nunca } \\
\text { puede ser aplicado en las primeras maniobras de masaje }\end{array}$ \\
\hline 8 & $\begin{array}{l}\text { En el masaje superficial se encuentra la técnica de } \\
\text { pellizcamiento }\end{array}$ \\
\hline 9 & $\begin{array}{l}\text { La vibración permite un mejor flujo sanguíneo y linfático, y una } \\
\text { distribución equilibrada del líquido entre los tejidos. }\end{array}$ \\
\hline 10 & $\begin{array}{l}\text { La técnica de sacudidas, dentro del masaje profundo se realiza } \\
\text { sobre brazos, piernas y cintura pélvica. }\end{array}$ \\
\hline
\end{tabular}

\section{Aplicación del Ciclo de mejora en el Aula}

\section{Relato resumido de las sesiones}

Tomamos nota de todo lo acontecido desde el primer momento utilizando el diario reflexivo del profesor, lo que nos ayudó bastante durante el desarrollo de la actividad. Comenzamos con la primera clase del modelo metodológico propuesto en la semana cuarta del segundo cuatrimestre, con la práctica 4. En esta sesión explicamos cuál sería el desarrollo de las siguientes sesiones. Hasta ese momento habíamos utilizado una metodología tradicional y les comunicamos que se iba a producir un cambio hacia 
otro modelo metodológico posible, lo que produjo cierta sorpresa en el alumnado. Una vez expuesto el sistema docente que se aplicaría en las siguientes clases prácticas, se resolvieron todas las dudas. Consideramos oportuno explicarles cuál era la finalidad de este modelo de enseñanza-aprendizaje, lo que generó cierta expectación que no se ha visto defraudada una vez concluido el periodo docente.

Una vez explicado la metodología a seguir en las siguientes sesiones y aclaradas las cuestiones planteadas, se pasó el pretest al alumnado para poder obtener la información necesaria sobre el nivel de sus conocimientos.

A continuación, se impartió el material teórico-práctico del tema correspondiente y se les explicó las técnicas que se iban a aplicar. Intentamos en todo momento crear un ambiente distendido y cordial siguiendo las sugerencias de Bain (2017).

A partir de este momento realizaron todas las prácticas por parejas, introduciendo un reto o problema a superar por cada alumno/a con su compañero/a, supervisados en todo momento por el profesor, comprobando que la metodología se aplicaba correctamente. Durante el desarrollo de la práctica los estudiantes fueron evaluados mediante una rúbrica elaborada previamente para esta cuestión, como veremos más adelante en la tabla 4. Una vez concluida esta fase de prácticas bajo supervisión, se realizó un debate grupal para reflexionar sobre todo lo acontecido, tomar notas de las posibles mejoras y corregir posibles defectos, con el fin de mejorar las posteriores dinámicas y el ciclo en general.

Conforme se avanzaba en las sesiones prácticas, se iba incrementando el volumen de trabajo. El buen ambiente y la motivación del alumnado facilitaban la carga de trabajo favoreciendo un clima relajado y participativo. A su manera y conforme a sus posibilidades, fueron aceptando el nuevo estilo de aprendizaje como algo positivo e innovador que formaba parte de una estrategia bien organizada y nada azarosa. 
Evaluación del aprendizaje de los estudiantes. Escaleras de aprendizaje

Como hemos mencionado con anterioridad, utilizamos una rúbrica para la evaluación del aprendizaje, en cada una de las sesiones prácticas. Con ella valorábamos la actitud del alumnado en todo momento, como desempeñaban la actividad y como afrontaban la tarea propuesta, enfrentándose al problema planteado por el profesor. Los alumnos/as tenían que resolver in situ, con los medios de los que disponían y con los conocimientos de los que previamente se le había dotado, cada uno de los retos planteados por el docente

Tabla 4. Sistema de rubrica para la evaluación individual de cada estudiante. Fuente: Elaboración propia

\begin{tabular}{|c|c|c|c|}
\hline \multicolumn{4}{|c|}{ RUBRICA EVALUACIÓN DE PRACTICA } \\
\hline CALIFICACIÓN & ACTITUDES & DESEMPEÑO DE ACTIVIDAD & TAREA PROPUESTA \\
\hline $\begin{array}{c}\text { MUY BIEN } \\
\text { (3) }\end{array}$ & $\begin{array}{l}\text { - Muy buena } \\
\text { disposición } \\
\text { - Muy buena } \\
\text { conducta }\end{array}$ & $\begin{array}{l}\text { - } \text { Posee grandes conocimien- } \\
\text { tos sobre la materia } \\
\text { - } \\
\text { Analiza muy bien sus } \\
\text { resultados } \\
\text { - } \text { Maneja el material muy bien } \\
\text { - } \text { Concluye muy bien }\end{array}$ & $\begin{array}{l}\text { - Cumple con } \\
\text { las tareas } \\
\text { perfectamente }\end{array}$ \\
\hline $\begin{array}{l}\text { BIEN } \\
(2)\end{array}$ & $\begin{array}{l}\text { - } \text { Buena } \\
\text { disposición } \\
\text { - } \text { Buena } \\
\text { conducta }\end{array}$ & $\begin{array}{l}\text { - } \text { Posee algunos conocimien- } \\
\text { tos sobre la materia } \\
\text { - } \\
\text { - } \\
\text { - Manaliza bien los resultados bien el material } \\
\text { - } \\
\text { Concluye bien }\end{array}$ & $\begin{array}{l}\text { - Cumple con las } \\
\text { tareas de forma } \\
\text { simple }\end{array}$ \\
\hline $\begin{array}{l}\text { SUFICIENTE } \\
\text { (1) }\end{array}$ & $\begin{array}{l}\text { - } \text { Poca } \\
\text { disposición } \\
\text { - } \text { Con- } \\
\text { ducta poco } \\
\text { adecuada }\end{array}$ & $\begin{array}{l}\text { - } \text { Posee escasos conocimien- } \\
\text { tos sobre la materia } \\
\text { - } \text { Analiza muy poco los } \\
\text { resultados } \\
\text { - } \text { Maneja mal el material } \\
\text { - } \\
\text { No concluye } \\
\text { adecuadamente }\end{array}$ & $\begin{array}{l}\text { - Cumple con las } \\
\text { tareas inade- } \\
\text { cuadamente }\end{array}$ \\
\hline
\end{tabular}

Jornadas de Formación e Innovación Docente del Profesorado | № 2 (2019) Esta obra se distribuye con la licencia Creative Commons Reconocimiento-NoComercial-SinObraDerivada Internacional (CC BY-NC-ND 4.0.) 


\begin{tabular}{|c|c|c|c|}
\hline $\begin{array}{c}\text { NO SUFICIENTE } \\
(0)\end{array}$ & $\begin{array}{l}\text { - No tiene } \\
\text { disposición } \\
\text { - Mala } \\
\text { conducta }\end{array}$ & $\begin{array}{l}\text { - No dispone de } \\
\text { conocimientos. } \\
\text { - } \text { Falta de analice en los } \\
\text { resultados } \\
\text { - } \text { Maneja el material } \\
\text { inadecuadamente } \\
\text { - No concluye }\end{array}$ & $\begin{array}{l}\text { - No cumple con } \\
\text { las tareas de } \\
\text { ninguna forma }\end{array}$ \\
\hline
\end{tabular}

En la siguiente figura mostramos el grado de satisfacción de los estudiantes dentro del proceso de aprendizaje. Los aspectos que recogía esta valoración eran el ambiente, los compañeros, las instalaciones y el personal docente y administrativo. Los posibles valores oscilaban desde $25 \mathrm{a}$ 100 y los resultados nos muestran que el mayor porcentaje de alumnado valoró cada una de las dimensiones analizadas con los valores de 75 o 100, tal como se puede ver de manera pormenorizada en la figura 4.

\section{Valoracion de Satisfacción}

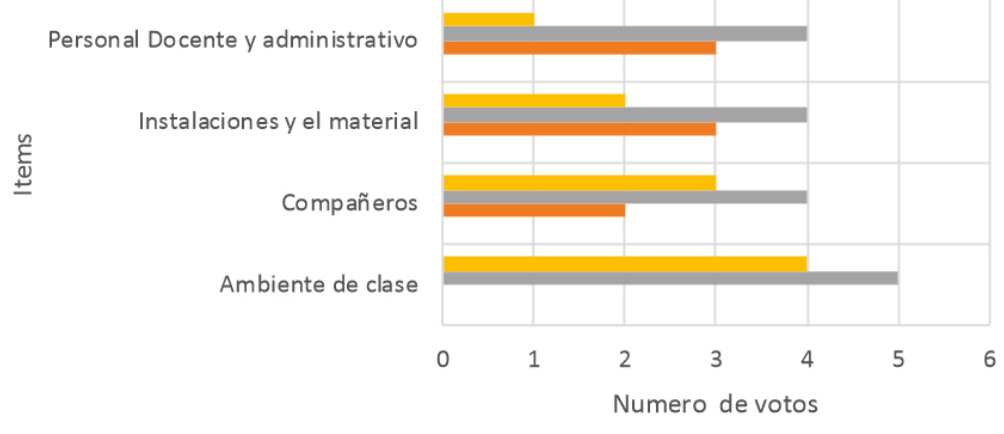

$\square 100=75 \square 50 \square 25$

Figura 4. Evaluaciones de satisfacción de los estudiantes. Fuente: Elaboración propia.

Para la evaluación completa del aprendizaje elaboramos varios cuestionarios en Socrative, uno por cada tema, con un test inicial (pretest) y un test final (postest), de este 
modo hemos podido conocer la evolución de cada estudiante. Como instrumento de evaluación utilizamos la escalera de aprendizaje tipo test, que constaba de un test con cinco preguntas y cuatro posibles respuestas cada una y otras cinco preguntas con respuestas de verdadero o falso. Estos cuestionarios iban cambiando, según las especificidades del tema a tratar. Los resultados de esta evaluación del aprendizaje se encuentran desglosados en la figura 5.

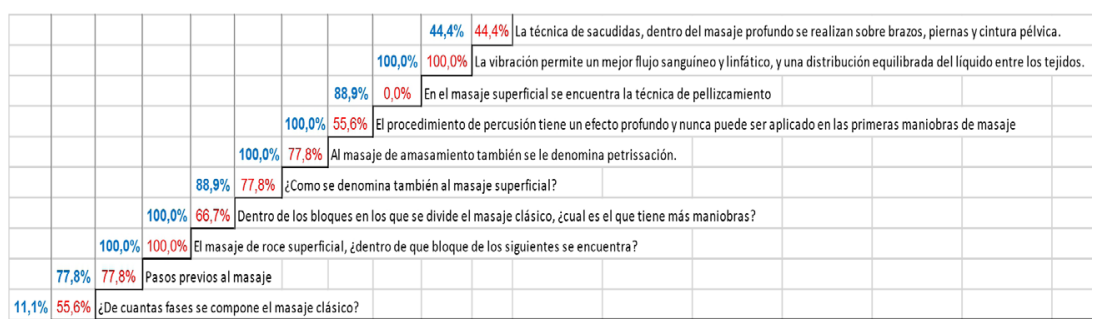

Figura 5. Escalera de Aprendizaje del Pretest y Postest de conocimientos en la asignatura de FG. Fuente: Elaboración propia.

Como se desprende de la anterior figura, el cambio producido desde los datos obtenidos en el test inicial (en color azul), sin contar con los conocimientos de la materia, y los datos que se reflejan después en el test posterior (en color naranja), tras recibir las materias teórico-prácticas en cuestión, son muy relevantes.

En la figura 6 quedan reflejados los cambios producidos en el aprendizaje desde el inicio hasta el final. Tomamos como ejemplo la casilla 1 (correspondiente a la puntuación media de aciertos totales en el test inicial y test final) con un valor de 6,56 frente a un valor final de un 8,11 . Estos datos reflejan los buenos resultados obtenidos en el proceso aprendizaje. 


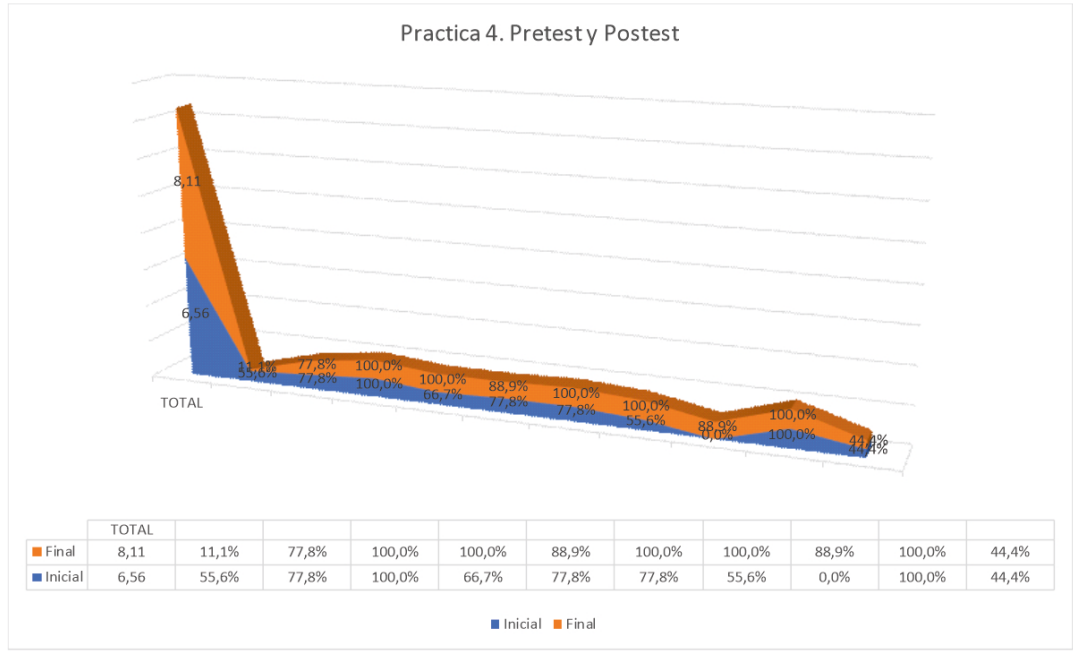

Figura 6. Gráfica, refleja el avance del proceso de aprendizaje. Fuente: Elaboración propia.

Y, por último, se ha realizado una encuesta de opinión sobre el CIMA, con objeto de tener una evaluación integral que nos permitiese extraer información sobre el proceso completo. La figura 7 refleja los datos de evaluación relacionados con las actividades, la metodología y el docente.

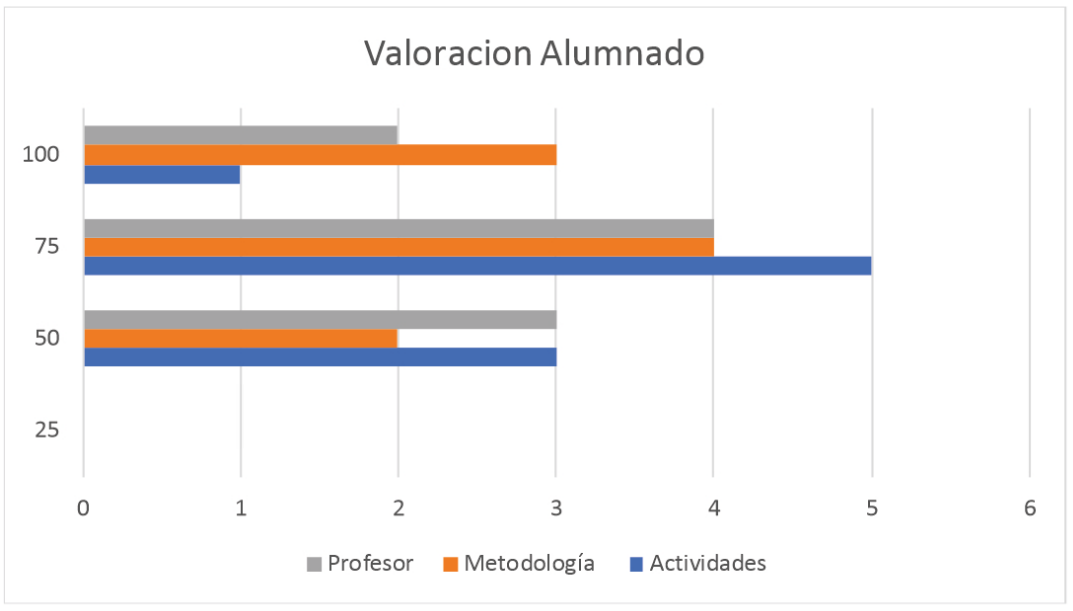

Figura 7. Valoración del alumnado. Fuente: Elaboración propia.

Jornadas de Formación e Innovación Docente del Profesorado | № 2 (2019) Esta obra se distribuye con la licencia Creative Commons Reconocimiento-NoComercial-SinObraDerivada 4.0 Internacional (CC BY-NC-ND 4.0.) 


\section{Evaluación del Ciclo de mejora en el Aula:}

\section{Cuestiones a mantener y cambios a introducir}

En el análisis de nuestro diseño y su aplicación, somos conscientes, como señala Porlán (2017), que lo que sucede en las clases es el resultado de la relación de varios elementos como son el modelo didáctico, las condiciones contextuales y proceso real de aprendizaje.

El modelo didáctico comienza evaluando el proceso desde su inicio: presentación, cuestionarios de conocimientos, desarrollo y exposición teórico-práctica, realización de las practicas por parejas (evaluadas con rubrica), reflexiones en grupo y varias evaluaciones más. Para desarrollar el trabajo de evaluación y autoevaluación del diseño, hemos utilizado varios instrumentos, como el diario del profesor, que aportan información valiosa y que favorecen la toma de decisiones e incluso la reflexión. También hemos pasado algunos cuestionarios a los estudiantes para analizar el proceso de enseñanza-aprendizaje propuesto en este diseño (encuestas de opinión, breves o largas). Así mismo, se realizó un cuestionario para la evaluación docente, incorporando algunas herramientas innovadoras como Kahoot y Socrative.

Con relación a las condiciones contextuales, tratamos de detectar las dificultades que han ido surgiendo en el aula y en nuestra labor docente, desde una actitud positiva proponiendo cómo superarlas, implicando a los estudiantes en todo el proceso e incluso a otros colegas.

En el transcurso del proceso de aprendizaje, durante la aplicación de nuestro CIMA, hemos encontrado algunas dificultades, que, gracias a la formación previa y la experiencia adquirida en nuestros anteriores CIMAS, han sido 
resueltas sin mayor dificultad y satisfactoriamente. Nos gustaría resaltar que nos hemos encontrado con un grupo de trabajo muy dispuesto, colaborador y entusiasta con las metodologías propuestas en estas sesiones.

Todo ello nos hace reflexionar sobre la importancia que ha tenido el desarrollo de las diversas estrategias en este CIMA, tales como han sido la metodología ABP, el aprendizaje basado en retos, el trabajo colaborativo, la gamificación y aula invertida, sin olvidar la importancia de los trabajos en equipo como design thinking, blended learning, motivación e implicación del aprendizaje y, por supuesto, los nuevos roles en educación. Por todo ello creemos que es oportuno seguir manteniendo esta forma de trabajo en nuestra docencia futura.

No obstante, creemos que debemos introducir los cambios de manera paulatina. Igualmente, sería muy beneficioso comenzar con modificar el sistema de evaluación, basado estrictamente en calificaciones, por un modelo metodológico donde prime la evaluación continua. En este esfuerzo, dada la gran diversidad y riquezas de nuevas metodologías de evaluación, estamos convencidos de encontrar otras técnicas que incorporaremos en ediciones posteriores del CIMA, convirtiéndose en nuevos desafios que revertirán sobre una mejor calidad docente y un mejor proceso de enseñanza-aprendizaje.

\section{Aspectos de la experiencia para incorporar en la práctica docente habitual.}

Con respecto de la experiencia desarrollada, sería muy interesante mantener y mejorar el diseño metodológico ejecutado, modificando todo lo necesario para conseguir optimizarlo dentro de lo posible. Como decíamos anteriormente, no deberíamos olvidar la reflexión continua de todas las partes, para seguir trabajando en una mejora docente de todo el proceso de enseñanza-aprendizaje. En este sentido, intentaremos modificar la manera 
de impartir los contendidos para que estos sean mucho más amenos, tomando como uno de los ejes principales, la participación y la colaboración. Después de años de experiencia tenemos muy claro la importancia que tiene la aplicación de metodologías innovadoras actuales en cualquier asignatura para conseguir mejores resultados en la enseñanza universitaria.

\section{Principios didácticos argumentados que han guiado la experiencia y que deben permanecer en el futuro.}

Para terminar con la valoración final y teniendo presente lo dicho anteriormente, creemos que hay algunos aspectos fundamentales que deben primar en nuestra docencia:

- Presentar la asignatura de forma amena para ir conociendo a los alumnos/as desde el primer momento.

- Empezar la primera unidad del temario realizando un cuestionario previo de conocimientos (pretest).

- Organizar la secuencia de actividades con la ayuda de un cronograma lo más realista posible teniendo presente las condiciones y el perfil del grupo.

- Tener presente la pregunta clave del mapa conceptual que nos ira dando paso a la introducción de las materias a través de los retos planteados.

- Estimular al estudiante para que puedan desarrollar su capacidad de reflexión y razonamiento.

- Emplear, desde el inicio, el diario reflexivo del profesor y llevar a cabo una buena planificación de todas las tareas.

- Desarrollar actividades en las cuales podamos desplegar todos los aspectos conceptuales, procedimentales y actitudinales del mapa de contenidos.

- Motivar el interés de los estudiantes por la materia.

- Promover la evaluación continua, desvinculando la calificación de la evaluación. 


\section{Referencias bibliográficas}

Bain, K. (2007). Lo que hacen los mejores profesores universitarios. Valencia: Publicaciones Universidad de Valencia.

Guerra, M. D. (2009). Opiniones de los Estudiantes de Enfermería sobre el Aprendizaje Basado en Problemas. Enfermería Global, 8 (3), 1-18.

Porlán, R. (2017). Enseñanza Universitaria, Cómo mejorarla. Madrid: Editorial Morata.

Sánchez, A. y Quintero, A. (2018). Socrative un recurso de respuesta inmediata en el Grado de Fisioterapia. Nuevas Pedagogías con Tecnologías emergentes, Madrid: Dykinson 